PROCEEDINGS OF THE

AMERICAN MATHEMATICAL SOCIETY

Volume 129, Number 2, Pages 585-592

S 0002-9939(00)05791-9

Article electronically published on August 28, 2000

\title{
UNIQUE ERGODICITY ON COMPACT HOMOGENEOUS SPACES
}

\author{
BARAK WEISS
}

(Communicated by Michael Handel)

\begin{abstract}
Extending results of a number of authors, we prove that if $U$ is the unipotent radical of an $\mathbb{R}$-split solvable epimorphic subgroup of a real algebraic group $G$ which is generated by unipotents, then the action of $U$ on $G / \Gamma$ is uniquely ergodic for every cocompact lattice $\Gamma$ in $G$. This gives examples of uniquely ergodic and minimal two-dimensional flows on homogeneous spaces of arbitrarily high dimension. Our main tools are the Ratner classification of ergodic invariant measures for the action of a unipotent subgroup on a homogeneous space, and a simple lemma (the 'Cone Lemma') about representations of epimorphic subgroups.
\end{abstract}

In his 1972 paper [F] Hillel Furstenberg proved that the horocycle flow on the unit tangent bundle to any compact Riemann surface is uniquely ergodic (i.e., admits a unique finite $U$-invariant Borel measure). This flow may be realized as the action of the subgroup $U$ of upper-triangular matrices in $L=\mathrm{SL}(2, \mathbb{R})$ on the quotient of $L$ by a cocompact lattice $\Lambda$, acting by

$$
u(\ell \Lambda)=(u \ell) \Lambda .
$$

Such an action is called a subgroup action on a homogeneous space. It follows from unique ergodicity that the action is minimal (i.e., any $U$-orbit is dense).

Various generalizations of Furstenberg's result were subsequently obtained by a number of authors (see $[\mathrm{V}],[\mathrm{Bo}],[\mathrm{EP},[\mathrm{D1}])$. In these generalizations what is proved is unique ergodicity of the action of $U$ on $L / \Lambda$, where $L$ is a real algebraic group, $U$ an algebraic subgroup which is unipotent (all its elements have all eigenvalues equal to 1 in a representation of $L$ as a matrix group; in other words all its elements are unipotent) and $\Lambda$ is a cocompact lattice. Typically in these results the dimension of the acting group $U$ may be quite large. In this note we generalize these results. For example, for any simple noncompact $L$, we obtain a 2-dimensional unipotent $U$ which acts uniquely ergodically on $L / \Lambda$ for any cocompact lattice $\Lambda$. On the other hand we show by examples that $L=\mathrm{SL}(3, \mathbb{R})$ does not contain any one-dimensional $U$ with this property.

The two essential ingredients we shall use are Marina Ratner's theorems regarding actions of a unipotent subgroup on a homogeneous space, and a simple result concerning epimorphic subgroups. Recall that Ratner's Measure Classification Theorem (see [R1], Theorem 1) asserts that if $L, \Lambda$ are as above, and $U$ is a connected subgroup of $L$ generated by unipotent elements, then for any $U$-invariant $U$-ergodic

Received by the editors April 22, 1999.

1991 Mathematics Subject Classification. Primary 22F30.

(C)2000 American Mathematical Society 
measure $\mu$ there are a connected subgroup $H$ and $x \in L / \Lambda$, such that $H$ contains $U$ and $\mu$ is an $H$-invariant measure supported on the closed orbit $H x$. Recall also that Ratner's Orbit-Closure Theorem (see [R2], Theorem A) asserts that if $L, \Lambda$ are as above, and $U$ is a connected subgroup of $L$ generated by unipotent elements, then for any $x \in L / \Lambda$ there exists a connected subgroup $H$ containing $U$ such that $\overline{U x}=H x$ and $H x$ supports an $H$-invariant probability measure. Ratner's results are in fact stronger (see [R3] for a survey).

Let $F<G$ be an inclusion of real algebraic groups. Recall that $F$ is epimorphic in $G$ if for any representation of real algebraic groups $\rho: G \rightarrow \mathrm{GL}(V)$, and any $v \in V$,

$$
\rho(F) v=v \Rightarrow \rho(G) v=v .
$$

In [Mo], Shahar Mozes proved that this representation-theoretic property has implications for invariant measures on homogeneous spaces. His theorem is the following:

Theorem 1 (Mozes). Let $F<G<L$ be an inclusion of real algebraic groups, where $G$ is generated by unipotent elements, and $F$ is epimorphic in $G$. Let $\Lambda$ be a discrete subgroup of $L$. Then any $F$-invariant probability measure on $L / \Lambda$ is $G$-invariant. In particular, if $G=L$, then the action of $F$ on $L / \Lambda$ is uniquely ergodic.

Note that in this result $\Lambda$ is not assumed to be cocompact. Our goal in this paper is to see to what extent the conclusion of Theorem 1 can be strengthened under the additional assumption that $\Lambda$ is cocompact. We should mention that by a result of Gregory A. Margulis (see Ma]) the cocompactness condition is satisfied whenever $L / \Lambda$ admits a uniquely ergodic action of a unipotent subgroup.

By a theorem of Frederic Bien and Armand Borel (see [BB], Theorem 2), for any algebraic group $G$ generated by unipotent elements and any epimorphic subgroup $F$, there is a subgroup $F^{\prime}$ of $F$ which is also epimorphic in $G$ and is of the form $F^{\prime}=T \cdot U$, where $U$ is unipotent and $T$ is an $\mathbb{R}$-split algebraic torus normalizing $U$. We now describe the setup for our results.

\section{BASIC ASSUMPTIONS}

Let $U<G<L$ be an inclusion of real algebraic groups, with $U$ unipotent and $G$ generated by unipotents. Assume that there is an $\mathbb{R}$-split torus $T$ such that $T$ normalizes $U$ and $T \cdot U$ is epimorphic in $G$. Let $\Lambda$ be a subgroup of $L$ such that $L / \Lambda$ is compact and carries a finite $L$-invariant measure.

Note that any simple noncompact $G$ contains a subgroup $U$ satisfying the Basic Assumptions, with $\operatorname{dim} U \leq 2$ (see [BB], $\S 5$ (b)).

These are our results:

Theorem 2. Let $U, G, L, \Lambda$ be as in the Basic Assumptions. Suppose also that $L=G$. Then the action of $U$ on $G / \Lambda$ is uniquely ergodic and minimal.

Theorem 3. Let $U, G, L, \Lambda$ be as in the Basic Assumptions. Suppose also that $G$ is normal in $L$. Then any $U$-invariant closed set, as well as any $U$-invariant measure, is also $G$-invariant.

Theorem 4. Let $U, G, L, \Lambda$ be as in the Basic Assumptions. Suppose also that the action of $G$ on $L / \Lambda$ is either minimal or uniquely ergodic. Then the action of $U$ is both minimal and uniquely ergodic. 
Theorems 2 3 and 4 all follow from the following:

Lemma 5. Let $U, G, L, \Lambda$ be as in the Basic Assumptions. Assume that $\Lambda$ is discrete. Let $H$ be a connected subgroup containing $U$ as in the conclusion of either Ratner's Measure Classification Theorem or Ratner's Orbit-Closure Theorem. Then for some $\ell \in L, G \subset \ell H \ell^{-1}$.

Lemma 5 follows in turn from the following simple Lemma, which is a variant of what we have called the 'cone lemma' (see [W], Lemma 1):

Lemma 6. Let $G$ be an algebraic group with no nontrivial rational characters, and let $T \cdot U$ be epimorphic in $G$, where $U$ is unipotent and $T$ is an $\mathbb{R}$-split torus normalizing $U$. Then for any representation (of real algebraic groups) $\rho: G \rightarrow$ $\mathrm{GL}(V)$, there is $t \in T$ such that for any $\rho(U)$-invariant vector $v, \rho\left(t^{n}\right) v$ converges to a $\rho(G)$-invariant vector as $n \rightarrow \infty$.

After receiving a preliminary version of this paper Nimish Shah pointed out to the author that the ideas used in proving Lemma 5 also have implications when $\Lambda$ is not assumed to be cocompact. To state them, recall that if $A$ is a semigroup in $L$ and $x \in L / \Lambda$, a trajectory $A x$ is said to be divergent if for every compact $K \subset L / \Lambda$ there exists a compact $C \subset G$ such that $a x \notin K$ whenever $a \in A-C$. Then the following holds:

Theorem 7 (Shah). Let $U<L$ be an inclusion of real algebraic groups, where $U$ is unipotent and $L$ is generated by the unipotent elements contained in it. Let $T$ be an $\mathbb{R}$-split torus in $L$, normalizing $U$, such that $T U$ is epimorphic in $L$. Then there is an open subsemigroup $T^{-} \subset T$ (a 'cone') such that for any lattice $\Lambda$ in $L$ and any $x \in L / \Lambda$, if $T^{-} x$ is not a divergent trajectory, then $\overline{U x}=L / \Lambda$.

Theorem 7 improves a result proved by S. G. Dani in 1985 (see [D2], Theorem 1.6).

The paper is organized as follows. First we prove Lemma 5 Then we deduce Theorems 2 3, 4 . We then show by an example that the Basic Assumptions alone do not guarantee that any $U$-invariant measure on $L / \Lambda$ is $G$-invariant. This explains why the additional hypotheses in our theorems are needed. We also explain why, for $L=\mathrm{SL}(3, \mathbb{R})$, no one-dimensional unipotent subgroup $U$ acts minimally on $L / \Lambda$ for every cocompact lattice $\Lambda$. We then sketch, for the reader's convenience, the proof of Lemma [6] and end by proving Theorem 7

Proof of Lemma國. Let $U, G, L, \Lambda, H$ be as in the statement of the Lemma. Thus $\overline{U \ell_{0} \Lambda}=H \ell_{0} \Lambda$ for some $\ell_{0} \in L$. Let $d=\operatorname{dim} H$ and let $\rho$ be the representation of $L$ on its $d$-dimensional Lie subalgebras. That is,

$$
\rho=\bigwedge_{1}^{d} \operatorname{Ad}: L \rightarrow \operatorname{GL}(V) \text { where } V=\bigwedge_{1}^{d} \operatorname{Lie}(L) .
$$

Let $v_{H}$ be a nonzero element in the one-dimensional subspace of $V$ corresponding to $\operatorname{Lie}(H)$.

We first claim that $\rho(L) v_{H}$ is closed in $V$. Indeed, it is proved in DMa, Theorem 3.4] that $\rho\left(\ell_{0} \Lambda \ell_{0}^{-1}\right) v_{H}$ is discrete. Hence if $\rho\left(\ell_{n}\right) v_{H} \rightarrow w$, then passing to a subsequence we can write, by the cocompactness of $\Lambda$,

$$
\ell_{n}=\tilde{\ell}_{n} \lambda_{n}, \text { where } \lambda_{n} \in \ell_{0} \Lambda \ell_{0}^{-1} \text { and } \tilde{\ell}_{n} \rightarrow \tilde{\ell} .
$$


Thus

$$
\rho\left(\lambda_{n}\right) v_{H} \rightarrow \rho\left(\tilde{\ell}^{-1}\right) w
$$

and, by discreteness,

$$
\rho\left(\tilde{\ell}^{-1}\right) w \in \rho\left(\ell_{0} \Lambda \ell_{0}^{-1}\right) v_{H}
$$

so $w \in \rho(L) v_{H}$.

Since $U \subset H, \rho(U)$ stabilizes the line through $v_{H}$ and since $U$ has no rational characters, $v_{H}$ is $\rho(U)$-invariant. Letting $T$ be as in the Basic Assumptions, we apply Lemma 6 to $\rho$ and to $v_{H}$. We obtain that for some $t \in T, \rho\left(t^{n}\right) v_{H} \rightarrow v_{0}$ where $v_{0}$ is $\rho(G)$-invariant. Since $\rho(L) v_{H}$ is closed there exists $\ell \in L$ such that $v_{0}=\rho(\ell) v_{H}$, that is, $v_{0}$ corresponds to the Lie algebra of $H^{\prime}=\ell H \ell^{-1}$, and $H^{\prime}$ is normalized by $G$. Since $T$ normalizes $U$, for any $n$ we have $U \subset t^{n} H t^{-n}$. This implies that also $U \subset H^{\prime}$.

We now claim that $G \subset H^{\prime}$. Indeed (replacing $H^{\prime}$ if necessary by the subgroup of $H^{\prime}$ generated by the unipotent elements in $H^{\prime}$ ), we can assume that $H^{\prime}$ is generated by unipotents. Therefore $H^{\prime}$ is algebraic (this follows from $\underline{S h}$, Lemma 2.9). Let $G_{0}=G \cap H^{\prime}$ (a normal subgroup of $G$ ) and let $\pi: G \rightarrow G / G_{0}$ be the natural quotient map. Since $H^{\prime}$ is algebraic, $\pi(T \cdot U)$ is epimorphic in $G / G_{0}$, and since $U \subset H^{\prime}, \pi(T \cdot U)=\pi(T)$ is an algebraic torus. Since proper toral subgroups are never epimorphic (see [BHM], Theorem 2) this implies that $G / G_{0}=\pi(T)$. However, $G / G_{0}$ is generated by unipotents since $G$ is, whereas $\pi(T)$ consists of semisimple elements. Thus $G / G_{0}$ is trivial, whence $G \subset H^{\prime}$.

\section{Deduction of Theorems 2, 3, 4}

First we claim that in proving the theorems we may assume that $\Lambda$ is discrete. For this we use an argument of Dave Witte (see [Wi]). Let $L_{0}$ be the Zariski closure of $\Lambda$. The space $L / L_{0}$ is the support of an $L$-invariant finite measure, namely the projection of the $L$-invariant measure on $L / \Lambda$. From Dani's version of the Borel density theorem (see $\mathrm{Wi}$, Theorem 4.2) it follows that $L_{0}$ contains an algebraic subgroup $L_{1}$ which is normal and cocompact in $L$. Since $G$ is generated by unipotents, the projection of $G$ onto $L / L_{1}$ is trivial, and therefore $G<L_{1}$. In particular $G<L_{0}$. Thus replacing $L$ by $L_{0}$ we may assume that $\Lambda$ is Zariski dense in $L$. Let $\Lambda^{0}$ be the connected component of the identity in $\Lambda$. Then $\Lambda$ normalizes $\Lambda^{0}$ and by Zariski density, so does $L$. We can now quotient out by $\Lambda^{0}$; this means that the map

$$
L / \Lambda \rightarrow\left(L / \Lambda^{0}\right) /\left(\Lambda / \Lambda^{0}\right), \quad \ell \Lambda \mapsto \ell \Lambda^{0}\left(\Lambda / \Lambda^{0}\right)
$$

is a homeomorphism which intertwines the action of $G$ on $L / \Lambda$ with the action of $G /\left(G \cap \Lambda^{0}\right)$ on $\left(L / \Lambda^{0}\right) /\left(\Lambda / \Lambda^{0}\right)$. Since $\Lambda / \Lambda^{0}$ is discrete, this proves our claim.

Suppose $G$ is normal in $L$. Given any orbit-closure $\overline{U x}$ (respectively, a $U$ invariant $U$-ergodic measure $\mu$ ), we get from Ratner's Orbit-Closure Theorem (resp., Ratner's Measure Classification Theorem) a subgroup $H$ such that $\overline{U x}=H x$ (respectively, $U \subset H$ and $\mu$ is $H$-invariant). From Lemma 5 we get that $G \subset H^{\prime}=$ $\ell H \ell^{-1}$, and since $G$ is normal, this implies that $G \subset H$. This proves Theorem 3 Theorem 2 is a special case. 
Now let us prove Theorem 4 . It follows from Ratner's theorems that for groups generated by unipotents, minimality and unique ergodicity are equivalent properties. So it will suffice to prove that if the action of $G$ is minimal, so is the action of $U$.

Let $x \in L / \Lambda$ and $\overline{U x}=H x$. Let $H^{\prime}=\ell H \ell^{-1}$ be as in Lemma 5. Then

$$
\overline{U x}=\overline{H x}=\overline{\ell^{-1} H^{\prime} \ell x}=\ell^{-1} \overline{H^{\prime} \ell x} \supset \ell^{-1} \overline{G \ell x}=L / \Lambda .
$$

Therefore the action of $U$ is also minimal.

\section{EXAMPLES}

1. Let $L=\mathrm{SL}(3, \mathbb{R})$,

$$
G_{1}=\left\{\left(\begin{array}{ccc}
* & * & 0 \\
* & * & 0 \\
0 & 0 & 1
\end{array}\right)\right\} \cap L
$$

and

$$
U=\left\{\left(\begin{array}{lll}
1 & t & 0 \\
0 & 1 & 0 \\
0 & 0 & 1
\end{array}\right): t \in \mathbb{R}\right\} .
$$

It is possible to find a cocompact lattice $\Lambda$ in $L$ such that $\Lambda \cap G_{1}$ is a lattice in $G_{1}$ (such an example was communicated to me by Mary Rees). Now let

$$
g=\left(\begin{array}{lll}
1 & 0 & 0 \\
0 & 1 & 0 \\
0 & 1 & 1
\end{array}\right)
$$

Then $g U g^{-1}=U$ and $G_{2}=g G_{1} g^{-1} \neq G_{1}$. It is easy to see that both $U, G_{1}, L, \Lambda$ and $U, G_{2}, L, \Lambda$ satisfy the Basic Assumptions, and (e.g. using the main result of $[\mathrm{F}]$ ) that $\overline{U \Lambda}=G_{1} \Lambda$. In particular, $\overline{U \Lambda} \neq G_{2} \Lambda$. Letting $\mu$ be the $G_{1}$-invariant measure on $\overline{G_{1} \Lambda}$ and setting $G=G_{2}$ gives a counterexample to the following statement, which is analogous to Theorem 1 Under the Basic Assumptions, any $U$-invariant measure on $L / \Lambda$ is $G$-invariant. This shows that some additional assumption on $G$, as in Theorems 2, 34 is required.

2. Again let $L=\mathrm{SL}(3, \mathbb{R})$. Let $G_{3}$ be the subgroup of $L$ whose Lie algebra is generated by

$$
\left(\begin{array}{ccc}
1 & 0 & 0 \\
0 & 0 & 0 \\
0 & 0 & -1
\end{array}\right),\left(\begin{array}{lll}
0 & 1 & 0 \\
0 & 0 & 1 \\
0 & 0 & 0
\end{array}\right),\left(\begin{array}{lll}
0 & 0 & 0 \\
1 & 0 & 0 \\
0 & 1 & 0
\end{array}\right) .
$$

An example communicated to me by A. Rapinchuk shows that one may find a cocompact lattice $\Lambda$ in $L=\operatorname{SL}(3, \mathbb{R})$ such that $G_{3} \cap \Lambda$ is a lattice in $G_{3}$. Since, by Jordan canonical form, any one-parameter unipotent subgroup of $L$ is conjugate either to a subgroup of $G_{3}$ or to a subgroup of $G_{1}$, we have that no one-parameter unipotent subgroup of $L$ acts uniquely ergodically or minimally on $L / \Lambda$ for every cocompact lattice $\Lambda$ in $L$. 
Proof of Lemma 6. Let $W$ be the subspace of $V$ consisting of $\rho(U)$-invariant vectors. Then $v \in W$ and $W$ is $\rho(T)$-invariant since $T$ normalizes $U$. Since $T$ is an $\mathbb{R}$-split torus, we can write

$$
W=\bigoplus_{\chi \in \Psi} W_{\chi},
$$

where $\Psi$ is a subset of the set of rational characters on $T$ and

$$
\forall w \in W_{\chi}, t \in T, \quad \rho(t) w=\chi(t) w .
$$

Denote the trivial character by 0 and for $\chi \in \Psi$ let $d \chi$ denote its derivative, which is just a linear functional on $\operatorname{Lie}(T)$. Write

$$
v=w_{0}+\sum_{\chi \in \Psi-\{0\}} w_{\chi}
$$

Since $T \cdot U$ is epimorphic in $G, w_{0}$ is $\rho(G)$-invariant. We will prove that for some $t \in T, \rho\left(t^{n}\right) v \rightarrow w_{0}$ by showing that there exists $t$ such that

$$
d \chi(t)<0 \text { for all } \chi \in \Psi-\{0\} .
$$

For this it suffices to show that 0 is not contained in the convex hull (over $\mathbb{R}$ ) of $\{d \chi: \chi \in \Psi-\{0\}\}$. If we had constants $a_{\chi} \in \mathbb{R}_{+}$such that

$$
\sum_{\chi \in \Psi-\{0\}} a_{\chi} d \chi=0, \quad \sum a_{\chi}=1,
$$

then using the fact that the derivatives of the rational characters of $T$ form a discrete $\mathbb{Z}$-module in $\operatorname{Lie}(T)^{*}$ it follows (see $[\mathrm{W}]$ for more details) that there are $b_{\chi} \in \mathbb{Z}_{+}$, not all zero, such that

$$
\sum_{\chi \in \Psi-\{0\}} b_{\chi} d \chi=0
$$

Consider then the action of $G$ on the vector

$$
w=\bigotimes_{\chi \in \Psi-\{0\}} w_{\chi}^{\otimes b_{\chi}}
$$

in the space $\otimes_{1}^{d} V$, where $d=\sum b_{\chi}$. Then it follows from (2) that $w$ is invariant under the action of $\rho_{0}(T \cdot U)$ and hence of $\rho_{0}(G)$, where $\rho_{0}=\bigotimes_{1}^{d} \rho$. Thus the line through each $w_{\chi}$ is invariant under $\rho(G)$. However, since $G$ has no rational characters, this implies that each $w_{\chi}$ is $\rho(G)$-invariant, which implies that $\Psi=$ $\{0\}$.

Proof of Theorem 7. The proof sketched here is somewhat different from the one communicated to the author by Nimish Shah.

Notice that the proof of Lemma 6 in fact implies a stronger statement: for any representation $\rho: G \rightarrow \operatorname{GL}(V)$ there is an open subsemigroup $T^{-} \subset T$ such that for any $\rho(U)$-invariant vector $v \in V$ and any sequence $\left\{t_{n}\right\}$ with $t_{n} \in T^{-}$and $t_{n} \rightarrow \infty$ (i.e., $t_{n}$ eventually leaves every compact subset of $G$ ), the sequence $\rho\left(t_{n}\right) v$ tends to a $\rho(G)$-invariant vector. To see this take

$$
T^{-}=\{t \in T: d \chi(t)<0 \text { for all } \chi \in \Psi-\{0\}\} .
$$


Arguing as in the proof of (1) in Lemma $\left[\right.$ shows that $T^{-}$is non-empty and satisfies the claim. So now let us take for $T^{-}$the semigroup corresponding to the natural action of $L$ on

$$
\bigoplus_{k=1}^{n} \bigwedge^{k} \operatorname{Lie}(L)
$$

Let $x=\ell_{0} \Lambda$, and suppose that $T^{-} x$ is not divergent; that is, there exists a compact $K \subset L$ and a sequence $t_{n} \in T^{-}$such that $t_{n} \rightarrow \infty$ and $t_{n} \ell_{0} \in K \Lambda$. Thus $t_{n} \in K \Lambda \ell_{0}^{-1}$. Let $\overline{U x}=H x$ and consider again the representation $\rho: L \rightarrow \operatorname{GL}(V)$ and the vector $v_{H} \in V$ as in the proof of Lemma 5. Notice that this representation is contained as a summand in (3).

Arguing as in the second paragraph of the proof of Lemma 5 we see that $\rho\left(K \Lambda \ell_{0}^{-1}\right) v_{H}$ is closed. On the other hand we know that $\rho\left(t_{n}\right) v_{H}$ tends to a $\rho(L)$ invariant vector as $n \rightarrow \infty$. This implies as in the proof of Lemma[5t that a conjugate $H^{\prime}$ of $H$ contains $U$ and is normalized by $L$. Again as in the proof of Lemma 5 we get that $L \subset H^{\prime}$.

\section{ACKNOWLEDGEMENTS}

I would like to thank Nimish Shah for a formulation and proof of Theorem 7 as well as additional comments on an earlier version of this paper, to Mary Rees and Andrei Rapinchuk for information on lattices used in the examples, and to the referee for helpful remarks.

\section{REFERENCES}

[BHM] A. Bialinicki Birula, G. Hochschild and G. D. Mostow, Extensions of Representations of Algebraic Linear Groups, Am. J. of Math. 85 (1963) 131-144. MR 27:5871

[BB] F. Bien and A. Borel, Sous-groupes epimorphiques de groupes lineaires algebrique I, C. R. Acad. Sci. Paris, bf t. 315 (1992) Serie I, 649-653. MR 93i:20048

[Bo] R. Bowen, Weak Mixing and Unique Ergodicity on Homogeneous Spaces, Isr. J. of Math. 23 (1976) 267-273. MR 53:11016

[D1] S. G. Dani, Bernoullian Translations and Minimal Horoshperes on Homogeneous Spaces, J. Ind. Math. Soc. 40 (1976) 245-284. MR 57:585

[D2] S. G. Dani, Divergent trajectories of flows on homogeneous spaces and Diophantine approximation, J. Reine Angew. Math. 359 (1985) 55-89. MR 87g:58110a

[DMa] S. G. Dani and G. A. Margulis, Limit Distributions of Orbits of Unipotent Flows and Values of Quadratic Forms, Advances in Soviet Mathematics, 16, Part 1, (1993) 91-137. MR 95b:22024

[EP] R. Ellis and W. Perrizo, Unique Ergodicity of Flows on Homogeneous Spaces, Isr. J. of Math. 29 (1978) 276-284. MR 57:12774

[F] H. Furstenberg, The Unique Ergodicity of the Horocycle Flow, Recent Advances in Topological Dynamics, A. Beck (ed.), Springer Verlag Lecture Notes 318 (1972) 95-115. MR 52:14149

[Ma] G. A. Margulis, Compactness of Minimal Closed Invariant Sets of Actions of Unipotent Groups, Geom. Ded. 37 (1991) 1-7. MR 92f:22018

[Mo] S. Mozes, Epimorphic Subgroups and Invariant Measures, Ergodic Theory and Dynamical Systems, Vol. 15, Part 6 (1995) 1207-1210. MR 96m:58143

[R1] M. Ratner, On Raghunathan's Measure Conjecture, Ann. Math. 134 (1991) 545-607. MR 93a:22009

[R2] M. Ratner, Raghunathan's Topological Conjecture and Distribution of Unipotent Flows, Duke J. of Math. 63 (1991) 235-280. MR 93f:22012

[R3] M. Ratner, Invariant Measures and Orbit Closures for Unipotent Actions on Homogeneous Spaces, Geometric and Functional Analysis, 4 (1994) 236-257. MR 95c:22018 
[Sh] N. A. Shah, Uniformly Distributed Orbits of Certain Flows on Homogeneous Spaces, Math. Ann. 289 (1991) 315-334. MR 93d:22010

[V] W. A. Veech, Unique Ergodicity of Horospherical Flows, Am. J. of Math. 99 no. 4 (1977) 827-859. MR 56:5788

[W] B. Weiss, Finite Dimensional Representations and Subgroup Actions on Homogeneous Spaces, Israel J. of Math. 106 (1998) 189-207. MR 99g:22017

[Wi] D. Witte, Measurable Quotients of Unipotent Translations on Homogeneous Spaces, Trans. AMS 345 no. 2 (1994) 577-594. MR 95a:22005

Department of Mathematics, State University of New York at Stony Brook, Stony Brook, New York 11794

E-mail address: barak@math.sunysb.edu 